\section{Epidemiologia das atividades \\ físicas praticadas no tempo de \\ lazer por adultos do Sul do Brasil}

\section{Epidemiology of leisure-time physical activities among adults from Southern Brazil}

\section{Samuel C. Dumith}

\section{Marlos R. Domingues}

\section{Denise P. Gigante}

Programa de Pós-Graduação em Epidemiologia, Universidade Federal de Pelotas, Brasil.

Agradecimentos: O primeiro autor agrade à CAPES (Coordenação de Aperfeiçoamento de Pesso al de Nível Superior), da qual foi bolsista durante a coleta de dados, e ao CNPq (Conselho Nacional de Desenvolvimento Científico e Tecnológico), do qual foi bolsista durante a redação deste artigo. Correspondência: Samuel C. Dumith. Rua 6, n. 163, Jardim do Sol, Rio Grande, RS, Brasil CEP 96216-090. E-mail: scdumith@yahoo.com.br

\section{Resumo}

Objetivo: Descrever os tipos de atividades físicas de lazer praticadas, bem como analisar o perfil de seus praticantes. Métodos: Pesquisa transversal de base-populacional com indivíduos de 20 anos ou mais, residentes na cidade de Pelotas, RS. O desfecho foi o tipo de atividade física de lazer praticada na semana anterior à entrevista. A associação bruta e ajustada com características demográficas, socioeconômicas e com o índice de massa corporal foi feita mediante testes qui-quadrado e regressão de Poisson, respectivamente. Resultados: Ao todo, foram entrevistados 3.136 indivíduos, dos quais $1.239(40 \%)$ relataram praticar alguma atividade física de lazer, constituindo-se no denominador deste estudo. A grande maioria (91\%) relatou praticar apenas uma atividade física. A modalidade mais praticada foi a caminhada, referida por $57 \%$ daqueles que estão engajados em alguma atividade. A seguir, as modalidades mais frequentes foram: futebol (14\%), bicicleta (13\%), musculação (8\%) e ginástica $(6 \%)$. A frequência semanal teve mediana de 3 dias, enquanto a mediana da duração diária foi de 60 minutos. Mais da metade (56\%) referiu que faz atividade física numa intensidade moderada ou vigorosa. No entanto, apenas $30 \%$ atingiram a recomendação proposta para adultos e idosos. Quando considerado o tempo de prática, $53 \%$ praticam atividade física há mais de seis meses. O motivo mais comum foi por razões de saúde. Conclusões: Menos da metade da população investigada pratica alguma atividade física no lazer. A modalidade mais comum para todos os grupos examinados foi caminhada. Diferenças no perfil demográfico e socioeconômico foram observadas conforme o tipo de atividade física.

Palavras-chave: Atividade motora. Exercício. Esporte. Atividades de lazer. Epidemiologia. Promoção da saúde. Estudo transversal. Brasil. 


\section{Abstract}

Objectives: To describe types of leisure-time physical activities and to analyze the profile of their practitioners. Methods: Cross-sectional population-based survey comprising individuals aged 20 years or over, resident in Pelotas, Brazil. The outcome was the type of leisure physical activity performed in the week before the interview. Crude and adjusted associations with demographic and socioeconomic characteristics and with body mass index (BMI) were conducted through chi-square and Poisson regression, respectively. Results: Overall, 3,136 individuals were interviewed, of which 1,239 (40\%) reported performing some leisure-time physical activity, corresponding to the denominator of this study. The great majority $(91 \%)$ performed only one physical activity. Walking was the most practiced, being reported by $57 \%$ of those engaged in some physical activity. The following most frequent activities were: soccer $(14 \%)$, cycling (13\%), weight lifting (8\%), and gymnastics (6\%). Median of weekly frequency was 3 days, while median of daily duration was 60 minutes. More than a half (56\%) reported performing physical activity with moderate-to-vigorous intensity. However, only $30 \%$ achieved the guidelines for adults and elderly. When the period of practice was considered, $53 \%$ were engaged in leisure physical activity for more than six months. The most common motivation was health reasons. Conclusions: Less than half of the investigated population was engaged in leisure-time physical activity. Walking was the most frequent modality for every examined group. Differences in the demographic and socioeconomic profile were observed according to the type of physical activity.

Keywords: Motor activity. Exercise. Sport. Leisure activities. Epidemiology. Health promotion. Cross-sectional study. Brazil.

\section{Introdução}

Embora o número de investigações científicas sobre a prática de atividade física no lazer e fatores associados tenha aumentado substancialmente nos últimos anos, existem poucos dados sobre os tipos de atividades físicas que as pessoas praticam no seu tempo livre, bem como suas características e o perfil dos praticantes. O IPAQ (International Physical Activity Questionnaire), que consiste num dos instrumentos mais empregados para mensurar o nível de atividade física da população ${ }^{1}$, não determina em quais modalidades de atividade física as pessoas estão engajadas, assim como a maior parte dos instrumentos utilizados em pesquisas epidemiológicas no campo da atividade física

No Brasil, são raros os estudos populacionais que descrevem o tipo de atividade física. Dentre os poucos estudos encontrados, destaca-se um levantamento feito nas regiões Sudeste e Nordeste do país em 1996972; um estudo realizado com funcionários de uma universidade do Rio de Janeiro ${ }^{3}$; e um feito com gestantes da cidade de Pelotas, $\mathrm{RS}^{4}$. Outro estudo, feito em São Paulo, com um pequeno grupo de homens voluntários, também coletou informações sobre o tipo de atividade realizada ${ }^{5}$. O tipo de atividade física praticada por adolescentes já foi descrito também por um estudo populacional ${ }^{6}$.

Assim como num inquérito alimentar é importante avaliar que tipos de alimentos as pessoas estão ingerindo, considera-se relevante investigar quais são as modalidades de atividades físicas mais praticadas pela população, bem como conhecer o perfil de seus praticantes. Tais dados podem ser empregados na formulação de políticas públicas, tanto na implementação de campanhas para determinados grupos populacionais, quanto em intervenções para modificar o ambiente em que as pessoas vivem.

Desse modo, este estudo teve como objetivo descrever o perfil das principais atividades físicas de lazer, em termos de frequência, duração, intensidade, tempo de prática e principal motivo para praticar atividade física; e analisar as características 
demográficas, socioeconômicas e de índice de massa corporal (IMC) dos praticantes das modalidades mais citadas.

\section{Metodologia}

O presente estudo possui delineamento transversal e foi realizado na cidade de Pelotas, RS. Pelotas é uma cidade da região sul do Brasil, com aproximadamente 320.000 habitantes, dos quais $93,5 \%$ residem na zona urbana. Para participar do estudo, os indivíduos deveriam ter 20 anos ou mais de idade e estar residindo na zona urbana do município no momento em que foi realizada a coleta dos dados. Foram excluídos os indivíduos institucionalizados (que viviam em asilos, hospitais ou presídios) e aqueles com incapacidade física e/ou mental para responder ao questionário. A coleta de dados ocorreu entre os meses de outubro e dezembro de 2005 .

O processo de seleção da amostra ocorreu em dois estágios. Primeiro, todos os setores censitários da cidade foram colocados em ordem crescente conforme a renda média mensal do chefe da família ${ }^{7}$, sendo selecionados aleatoriamente 120 setores censitários com probabilidade proporcional ao seu tamanho. Segundo, todos os domicílios dos setores amostrados foram listados, $\mathrm{e}$ uma média de 13 domicílios por setor foram selecionados, sistematicamente, totalizando 1.597 domicílios sorteados. Como havia em média 2,1 adultos (pessoas com 20 anos ou mais) em cada domicílio, a amostra final resultou em 3.353 indivíduos. Este número foi suficiente para atender às demandas amostrais de um amplo estudo sobre saúde e comportamento da população pelotense ${ }^{8}$. Neste trabalho, foram analisados apenas os indivíduos que relataram ter realizado alguma atividade física de lazer na semana anterior à entrevista.

Todas as variáveis foram coletadas por meio de um questionário aplicado na forma de entrevistas face a face. As entrevistadoras recrutadas possuíam mais de 18 anos de idade e pelo menos nível médio de escolaridade. Foram treinadas durante 40 horas, mediante o ensino de técnicas de entrevista e de abordagem domiciliar, leitura e explicação do manual de instruções, processos de dramatização, e aplicação de um estudopiloto. Nenhuma delas estava ciente quanto aos objetivos e hipóteses da pesquisa. Um estudo-piloto em um setor não amostrado para a pesquisa foi realizado para verificar o desempenho em situação real de entrevista e realizar os últimos ajustes no instrumento. Para o controle de qualidade das entrevistas, um supervisor aplicou um questionário reduzido em $10 \%$ da amostra, selecionada de maneira aleatória.

A variável principal deste estudo consistiu na modalidade de atividade física que os indivíduos relataram praticar durante o seu tempo de lazer. Essa variável foi medida por meio de duas perguntas (uma fechada e uma aberta): a) "Desde < dia da semana passada>, o(a) Sr.(a) fez alguma atividade física por pelo menos 10 minutos seguidos durante o seu tempo livre?"; b) Se sim, “Qual (quais)?”. Dessa forma, foram consideradas apenas as atividades físicas de lazer praticadas nos sete dias anteriores à entrevista. Além do tipo de atividade física, coletaramse informações sobre a frequência (número de dias por semana), duração (minutos por dia), intensidade (leve, moderada, vigorosa) e principal motivo para praticar atividade física (pergunta aberta).

Para coletar as demais variáveis do estudo, foi utilizado um questionário padronizado e previamente testado. Foram coletadas informações sobre as seguintes variáveis: sexo (masculino/feminino); idade em anos completos (categorizada em 20 a 39, 40 a 59 e 60 ou mais); cor da pele coletada conforme as categorias do IBGE e posteriormente categorizada em branco/não-branco; situação conjugal (se morava com companheiro(a) ou sem companheiro(a)); escolaridade em anos completos de estudo (categorizada em 0 a 4, 5 a 8 e 9 ou mais); nível econômico conforme o instrumento da Associação Brasileira de Empresas de Pesquisa (ABEP) ${ }^{9}$; e índice de massa corporal (IMC), calculado com base no peso e altura auto-referidos, e categorizado em eutróficos (IMC $<25,0$ 
$\left.\mathrm{kg} / \mathrm{m}^{2}\right)$, com sobrepeso $\left(25,0\right.$ a $\left.29,9 \mathrm{~kg} / \mathrm{m}^{2}\right)$ e obesos (IMC $\geq 30 \mathrm{~kg} / \mathrm{m}^{2}$ ).

Quanto ao tratamento estatístico empregado, a entrada dos dados seguiu o processo de dupla digitação, com checagem automática de consistência e amplitude, no programa Epi-Info - versão 6.04. Para a análise dos dados, utilizou-se o pacote estatístico Stata - versão 9.2. Após os procedimentos descritivos (médias e proporções), analisou-se a distribuição das principais modalidades de atividade física de acordo com as variáveis estudadas, utilizando-se o teste qui-quadrado. Para a análise multivariável, elaborou-se um modelo composto por três níveis, sendo as variáveis ajustadas para aquelas do mesmo nível ou de níveis acima $^{10}$. No primeiro, entraram as variáveis demográficas (sexo, idade, cor da pele e situação conjugal). No segundo, as variáveis socioeconômicas (escolaridade e nível econômico). E no terceiro nível, o IMC. A análise ajustada foi feita por meio de regressão de Poisson, com variância robusta ${ }^{11}$, tendo sido levadas todas as variáveis e mantidas apenas aquelas com valor p menor que $0,2^{12}$. O nível de significância adotado foi de 5\% para testes bi-caudais.

O protocolo do estudo foi submetido e aprovado pelo Comitê de Ética da Faculdade de Medicina da Universidade Federal de Pelotas (UFPel). Além disso, todos os participantes da pesquisa assinaram um termo de consentimento livre e esclarecido.

\section{Resultados}

Dos 3.353 indivíduos selecionados para o estudo, foram entrevistados 3.136, correspondendo a uma taxa de resposta de $93,5 \%$. Dentre os 3.136 adultos entrevistados, 1.239 $(39,5 \%)$ relataram ter praticado alguma atividade física de lazer, correspondendo à amostra analisada no presente artigo. Destes 1.239 indivíduos, aproximadamente metade era do sexo feminino $(50,4 \%)$, tinha entre 20 e 39 anos de idade $(48,2 \%)$ e nove anos ou mais de escolaridade $(53,4 \%)$; a grande maioria era de cor branca (85,5\%); em torno de $60 \%$ moravam com companheiro(a); mais de um terço (38\%) pertencia às classes econômicas $\mathrm{A}$ ou $\mathrm{B}$; $\mathrm{e}$ $13 \%$ eram obesos (IMC $\geq 30 \mathrm{~kg} / \mathrm{m}^{2}$ ).

A Tabela 1 descreve as modalidades de atividade física de lazer em termos de prevalência (\%), frequência semanal (dias/sem), duração diária (min/dia) e duração semanal (min/sem). Salienta-se que a porcentagem total ultrapassa $100 \%$, pois um indivíduo poderia praticar mais de uma atividade física. Apesar disso, mais de 90\% dos entrevistados $(\mathrm{n}=1.129)$ praticaram apenas uma atividade física; duas, três e quatro modalidades diferentes foram praticadas por $7,4 \%(n=92), 1,1 \%(n=13)$ e $0,4 \%(n=$ 5) dos indivíduos, respectivamente, o que representa $9 \%$ da amostra analisada.

A atividade física mais frequente foi a caminhada, praticada por mais da metade dos indivíduos (57\%). A seguir, vieram o futebol (14\%), a bicicleta (13\%), a musculação (8\%), a ginástica (6\%) e a corrida (4\%). As demais atividades tiveram frequência inferior a $2 \%$ e, juntas, corresponderam a $8 \%$. A mediana da frequência semanal foi de 3 dias por semana, enquanto a duração diária e semanal tiveram medianas de $60 \mathrm{~min} /$ dia e $140 \mathrm{~min} /$ sem, respectivamente.

A Figura 1 ilustra a proporção de homens e mulheres dentre os praticantes das principais atividades físicas de lazer. Percebe-se que em atividades como futebol, bicicleta, musculação e corrida, os homens são maioria. Por outro lado, há mais mulheres praticando caminhada e ginástica.

Quando inquiridos sobre o principal motivo para praticar atividade física, observa-se que a motivação difere conforme o tipo de atividade praticada (Figura 2). Por exemplo, enquanto mais da metade (58\%) dos indivíduos que caminham relatam fazer isso por saúde, a maioria (63\%) dos que jogam futebol o faz por recreação. Outra constatação é que quase um terço (31\%) daqueles que pratica ginástica relatou que o principal motivo é a estética e/ou emagrecimento. Além disso, $10 \%$ dos que correm e $16 \%$ dos que fazem musculação informaram que praticam atividade física para melhorar o condicionamento. 
Tabela 1 - Frequência e duração das atividades físicas praticadas no tempo de lazer. Adultos ( $\geq 20$ anos) de Pelotas, RS, Brasil, $2005(\mathrm{~N}=1.239)$.

Table 1 - Frequency and duration of physical activities performed in leisure time. Adults ( $\geq 20$ years), Pelotas, Brazil, 2005 ( $N=$ $1,239)$.

\begin{tabular}{lcccc}
\hline Modalidade & $\mathrm{N}(\%)^{*}$ & Frequência (dias/sem) & Duração 1 (min/dia) & Duração 2 (min/sem) \\
\hline Caminhada & $706(57,0)$ & 3,9 & 52 & 203 \\
Futebol & $169(13,6)$ & 2,1 & 64 & 135 \\
Bicicleta & $160(12,9)$ & 4,4 & 57 & 271 \\
Musculação & $102(8,2)$ & 4,1 & 79 & 347 \\
Ginástica & $71(5,7)$ & 3,9 & 49 & 178 \\
Corrida & $54(4,4)$ & 4,2 & 59 & 264 \\
Dança & $22(1,8)$ & 2,9 & 104 & 248 \\
Alongamento & $21(1,7)$ & 4,1 & 36 & 138 \\
Artes marciais & $15(1,2)$ & 3,7 & 93 & 253 \\
Hidroginástica & $13(1,1)$ & 2,9 & 59 & 188 \\
Vôlei & $9(0,7)$ & 2,1 & 79 & 179 \\
Tênis & $8(0,7)$ & 3,8 & 95 & 354 \\
Natação & $8(0,7)$ & 3,0 & 64 & 203 \\
Basquete & $4(0,3)$ & 3,5 & 75 & 278 \\
Remo & $2(0,2)$ & 4,5 & 70 & 365 \\
Golfe & $2(0,2)$ & 3,0 & 120 & 360 \\
Bocha & $1(0,1)$ & 1,0 & 120 & 120 \\
\hline Média & - & $\mathbf{3 , 6}$ & $\mathbf{5 7}$ & $\mathbf{2 0 7}$ \\
\hline
\end{tabular}

* A soma ultrapassa $100 \%$ visto que um indivíduo poderia estar engajado em mais de uma modalidade de atividade física.

* The sum exceeds $100 \%$ because one individual could be engaged in more than one type of physical activity.

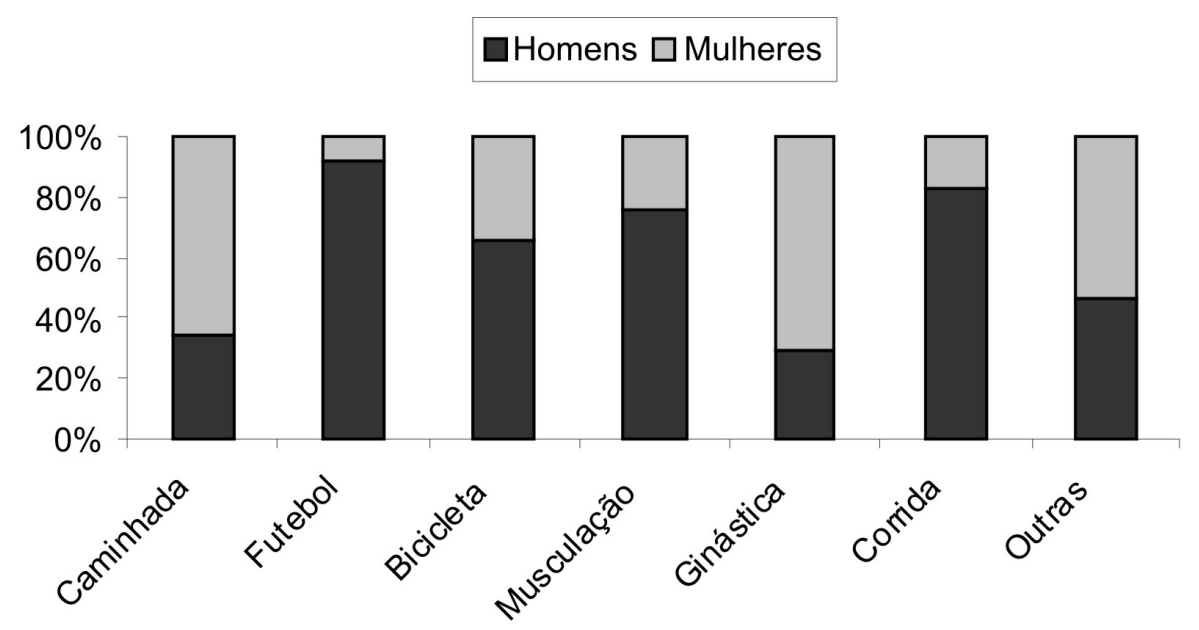

Tipo de AF

Figura 1 - Proporção de homens e mulheres conforme o tipo de atividade física (AF) de lazer praticada. Adultos ( $\geq 20$ anos) de Pelotas, RS, Brasil, 2005 ( $N=1.239$ ).

Figure 1 - Proportion of men and women according to the type of leisure-time physical activity. Adults ( $\geq 20$ years), Pelotas, Brazil, 2005 ( $N=1,239)$. 


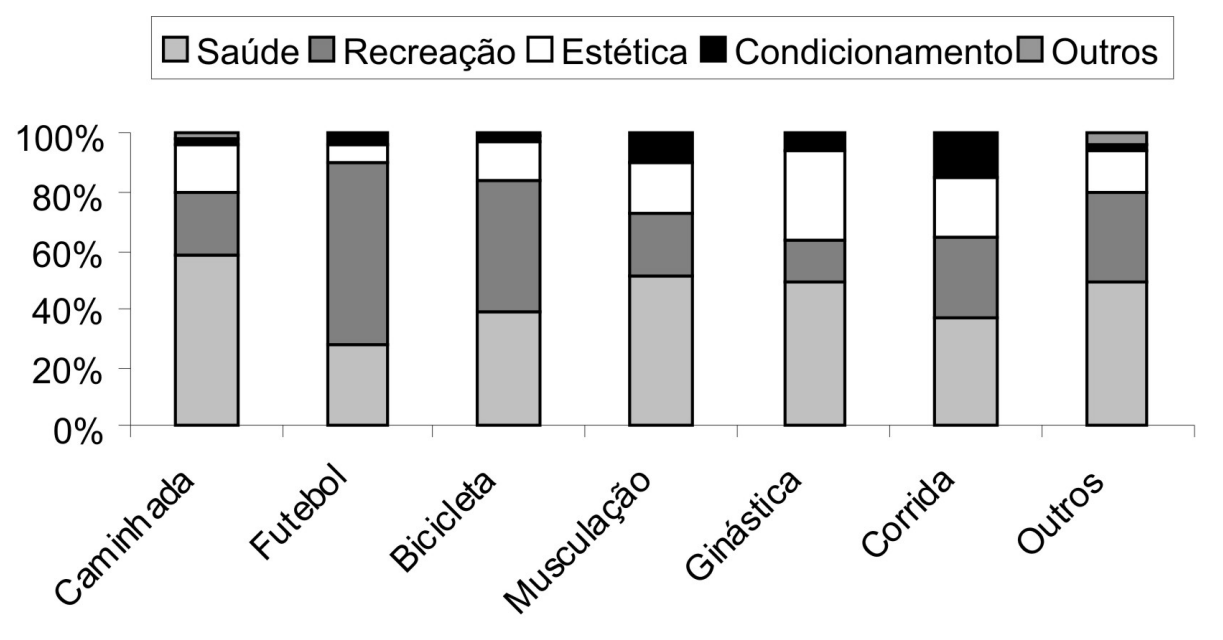

Tipo de AF

Figura 2 - Principal motivo para praticar atividade física (AF) de acordo com o tipo de AF praticada. Adultos ( $\geq 20$ anos) de Pelotas, RS, Brasil, 2005 ( $\mathrm{N}=1.239)$.

Figure 2 - Main reason for performing physical activity (PA) according to the type of PA. Adults $(\geq 20$ years), Pelotas, Brazil, $2005(N=1,239)$.

Na Tabela 2 estão descritas outras características dos praticantes das modalidades de atividade física de lazer. Observa-se que, enquanto menos de $10 \%$ dos que caminham também fazem outras atividades, entre os que praticam corrida, mais da metade está engajada em mais alguma atividade física. Com relação à intensidade, a proporção dos que se exercitam numa intensidade moderada a vigorosa variou de $49 \%$ (caminhada) a 78\% (corrida). Quando considerado o critério de $150 \mathrm{~min} / \mathrm{sem}$ de atividades moderadas ou $60 \mathrm{~min} / \mathrm{sem}$ de atividades vigorosas ${ }^{13}$, verifica-se que mais da metade dos que correm ou fazem musculação atinge tal recomendação; ao passo que menos de um terço dos que caminham, jogam futebol ou andam de bicicleta figura neste grupo. Salienta-se que a proporção de indivíduos que atingem o critério para ser considerado ativo leva em conta todo o conjunto de atividades praticadas no lazer, e não necessariamente apenas a que está sendo considerada na tabela. No entanto, menos de $10 \%$ relataram praticar mais de uma atividade física. Em análise adicional considerando o cumprimento das recomendações apenas pela atividade física analisada, os resultados foram basicamente os mesmos (dados não-apresentados). Pela última coluna da Tabela 2, nota-se que a maioria dos praticantes de cada modalidade pratica atividade física há pelo menos seis meses. Novamente, esses percentuais não significam que eles estejam necessariamente envolvidos em tais atividades há mais de seis meses, mas que são praticantes habituais de atividade física (qualquer) no seu tempo livre.

Com relação aos fatores associados às principais modalidades de atividade física de lazer, sexo, idade e escolaridade apresentaram associação com a maioria delas (Tabela 3). A caminhada foi a atividade mais frequente para todos os grupos examinados, sendo mais prevalente para as mulheres, idosos, indivíduos com pouca escolaridade e obesos. O futebol foi mais praticado pelos homens, adultos jovens e pelos indivíduos com mais escolaridade. Andar de bicicleta foi mais comum para os homens, adultos, indivíduos com companheiro(a), com menos escolaridade e mais pobres. A musculação foi mais praticada pelos homens, adultos jovens, indivíduos sem companheiro(a), de maior escolaridade e nível econômico. A prática de ginástica foi mais comum entre as mulheres, indivíduos 
Tabela 2 - Proporção de indivíduos que: praticam mais de uma atividade física (AF); praticam AF com intensidade moderada a vigorosa; que atingem o ponto de corte de $150 \mathrm{~min} / \mathrm{sem}$ de $A F$ moderada ou $60 \mathrm{~min} / \mathrm{sem}$ de AF vigorosa; e que praticam AF há mais de 6 meses; de acordo com o tipo de AF praticada. Adultos ( $\geq 20$ anos) de Pelotas, RS, Brasil, $2005(\mathrm{~N}=1.239)$.

Table 2 - Proportion of individuals engaged in more than one type of physical activity (PA); that perform moderate-tovigorous $P A$; that achieve the cut-off of $150 \mathrm{~min} / \mathrm{wk}$ of moderate $P A$ or $60 \mathrm{~min} / \mathrm{wk}$ of vigorous $P A$; and that have been engaged in PA for more than 6 months; according to the type of PA practiced. Adults ( $\geq 20$ years), Pelotas, Brazil, 2005 ( $N=1,239)$.

\begin{tabular}{lcccc}
\hline Tipo de AF & $\begin{array}{c}\text { Pratica mais } \\
\text { de 1 AF (\%) }\end{array}$ & $\begin{array}{c}\text { Intensidade moderada } \\
\text { a vigorosa (\%) }\end{array}$ & $\begin{array}{c}\text { Atinge critério } \\
\text { recomendado } \\
\text { de AF (\%) }\end{array}$ & $\begin{array}{c}\text { Pratica AF há mais } \\
\text { de 6 meses (\%) }\end{array}$ \\
\hline Caminhada & 9,1 & 49,4 & 27,2 & 53,4 \\
Futebol & 20,1 & 75,7 & 29,6 & 53,3 \\
Bicicleta & 16,3 & 51,9 & 31,9 & 49,4 \\
Musculação & 31,4 & 70,6 & 58,8 & 68,6 \\
Ginástica & 31,0 & 74,7 & 42,3 & 50,7 \\
Corrida & 55,6 & 77,8 & 55,6 & 81,5 \\
Outras & 31,1 & 60,2 & 37,9 & 58,3 \\
\hline Total & $\mathbf{8 , 9}$ & $\mathbf{5 6 , 4}$ & $\mathbf{3 0 , 5}$ & $\mathbf{5 3 , 0}$ \\
\hline
\end{tabular}

com maior escolaridade e pertencentes às classes econômicas mais altas. A corrida foi mais frequente entre os homens, adultos jovens, e indivíduos com maior escolaridade. A prática de outras atividades físicas (prevalência inferior a $2 \%$ ) foi maior entre os indivíduos sem companheiro(a) e com maior escolaridade e/ou nível econômico.

Os resultados da análise multivariável foram muito semelhantes aos encontrados na análise bruta (descrita no parágrafo acima) e, portanto, não estão apresentados. Não houve nenhum caso de confusão negativa, isto é, não o surgimento de uma associação ou aumento de um efeito na análise ajustada. As poucas alterações foram para as variáveis que atingiram um valor $p$ limítrofe na análise bruta (próximo de 0,05 ) e perderam a significância estatística na análise ajustada. Essa situação ocorreu para as seguintes associações: idade e bicicleta; situação conjugal e musculação; classe econômica e bicicleta ou outras atividades; e IMC e musculação.

\section{Discussão}

Neste trabalho, foi feito um levantamento das atividades físicas que as pessoas praticam no seu tempo de lazer e sobre as características das principais modalidades, bem como o perfil de seus praticantes. Não se encontrou nenhum outro estudo com o mesmo enfoque na literatura científica. Alguns poucos estudos, tanto no Brasil quanto no mundo, apenas coletaram o tipo de atividade física praticada e analisaram posteriormente o escore global de atividade física. Entretanto, muito pouco se conhece sobre a epidemiologia de cada modalidade e os seus adeptos.

De todos os indivíduos entrevistados na pesquisa ( $n=3.136), 40 \%(n=1.239)$ fizeram alguma atividade física no seu tempo livre, correspondendo ao denominador do presente estudo. Esse percentual está um pouco abaixo do que foi encontrado em uma investigação com funcionários de uma universidade do Rio de Janeiro, que obteve $46 \%{ }^{3}$. Contudo, no referido estudo, foram investigados apenas indivíduos entre $20 \mathrm{e}$ 60 anos e de um determinado padrão socioeconômico, o que pode ter superestimado essa prevalência. Um estudo populacional conduzido com adultos e idosos de Salvador (BA) encontrou que $37,5 \%$ praticavam atividade física de lazer numa semana habitual ${ }^{14}$. Outro estudo realizado em São 
Tabela 3 - Perfil dos praticantes de cada modalidade conforme características demográficas, socioeconômicas e índice de massa corporal (IMC).* Adultos ( $\geq 20$ anos) de Pelotas, RS, Brasil, 2005 ( $N=1.239$ ).

Table 3 - Profile of physical activity practitioners according to demographic and socioeconomic characteristics and body mass index (BMI).*Adults ( 220 years). Pelotas, Brazil, $2005(N=1,239)$.

\begin{tabular}{|c|c|c|c|c|c|c|c|c|}
\hline Variável & $\mathrm{N}$ & $\begin{array}{c}\text { Caminhada } \\
(\%)\end{array}$ & $\begin{array}{c}\text { Futebol } \\
(\%)\end{array}$ & $\begin{array}{c}\text { Bicicleta } \\
(\%)\end{array}$ & $\begin{array}{c}\text { Musculação } \\
\text { (\%) }\end{array}$ & $\begin{array}{c}\text { Ginástica } \\
\text { (\%) }\end{array}$ & $\begin{array}{c}\text { Corrida } \\
\text { (\%) }\end{array}$ & $\begin{array}{c}\text { Outras } \\
(\%)^{\dagger}\end{array}$ \\
\hline Sexo & & $P<0,001$ & $p<0,001$ & $p<0,001$ & $p<0,001$ & $p=0,001$ & $p<0,001$ & $p=0,531$ \\
\hline Masculino & 614 & 40,0 & 25,4 & 17,3 & 12,2 & 3,4 & 7,3 & 7,8 \\
\hline Feminino & 625 & 73,6 & 2,1 & 8,6 & 4,3 & 8,0 & 1,4 & 8,8 \\
\hline Idade (anos) & & $P<0,001$ & $p<0,001$ & $p=0,043^{\ddagger}$ & $p<0,001$ & $p=0,633$ & $p<0,001$ & $p=0,296$ \\
\hline $20-39$ & 597 & 41,2 & 21,9 & 12,4 & 12,2 & 6,4 & 6,5 & 9,6 \\
\hline $40-59$ & 435 & 66,4 & 8,3 & 15,6 & 6,0 & 5,3 & 3,5 & 6,9 \\
\hline$\geq 60$ & 207 & 82,6 & 1,0 & 8,7 & 1,5 & 4,8 & 0,0 & 7,7 \\
\hline Cor da pele & & 0,218 & 0,080 & 0,673 & 0,158 & 0,812 & 0,395 & 0,147 \\
\hline Branco & 1059 & 57,7 & 12,9 & 12,8 & 8,7 & 5,7 & 4,2 & 8,8 \\
\hline Não-branco & 180 & 52,8 & 17,8 & 13,9 & 5,6 & 6,1 & 5,6 & 5,6 \\
\hline Situação conjugal & & $p=0,812$ & $p=0,923$ & $p=0,002$ & $p=0,034^{\ddagger}$ & $p=0,967$ & $p=0,280$ & $p=0,025$ \\
\hline s/ companheiro(a) & 509 & 56,6 & 13,8 & 9,4 & 10,2 & 5,7 & 5,1 & 10,4 \\
\hline c/ companheiro(a) & 730 & 57,3 & 13,6 & 15,3 & 6,9 & 5,8 & 3,8 & 6,9 \\
\hline Escolaridade (anos) & & $p<0,001$ & $p<0,001$ & $p=0,001$ & $p<0,001$ & $p=0,010$ & $p=0,005$ & $p=0,014^{\ddagger}$ \\
\hline$\leq 4$ & 214 & 71,0 & 6,5 & 16,4 & 1,9 & 2,8 & 1,4 & 5,1 \\
\hline $5-8$ & 363 & 50,4 & 19,0 & 17,1 & 5,5 & 4,1 & 3,0 & 6,3 \\
\hline$\geq 9$ & 661 & 56,0 & 13,0 & 9,5 & 11,8 & 7,6 & 6,1 & 10,4 \\
\hline Classe econômica & & $p=0,803$ & $p=0,151$ & $p=0,048^{\ddagger}$ & $p<0,001$ & $p=0,011^{\ddagger}$ & $p=0,211$ & $p=0,041^{\ddagger}$ \\
\hline $\mathrm{A} / \mathrm{B}$ & 470 & 58,3 & 12,3 & 10,4 & 12,1 & 8,3 & 5,3 & 10,0 \\
\hline $\mathrm{C}$ & 518 & 56,4 & 15,4 & 13,7 & 5,4 & 4,3 & 4,4 & 8,5 \\
\hline $\mathrm{D} / \mathrm{E}$ & 243 & 56,4 & 14,4 & 16,5 & 6,2 & 4,1 & 2,5 & 4,5 \\
\hline $\operatorname{IMC}^{\S}\left(\mathrm{kg} / \mathrm{m}^{2}\right)$ & & $p=0,001$ & $p=0,169$ & $p=0,949$ & $p=0,049^{\ddagger}$ & $p=0,199$ & $p=0,164$ & $p=0,360$ \\
\hline$<25$ & 599 & 51,9 & 14,4 & 12,7 & 10,4 & 7,0 & 5,3 & 7,4 \\
\hline 25,0 a 29,9 & 459 & 58,6 & 14,6 & 13,3 & 6,8 & 5,0 & 4,1 & 9,8 \\
\hline$\geq 30$ & 157 & 68,2 & 8,9 & 13,4 & 5,7 & 3,8 & 1,9 & 8,3 \\
\hline Total & 1.239 & 57,0 & 13,6 & 12,9 & 8,2 & 5,7 & 4,4 & 8,3 \\
\hline
\end{tabular}

* Os valores percentuais se referem ao $\mathrm{N}$ de cada linha, e somam mais de $100 \%$ pois o indivíduo poderia praticar mais de uma modalidade de atividade física.

* Percentage values correspond to $\mathrm{N}$ of each row, and the sum exceeds $100 \%$ because one could be engaged in two or more types of physical activity.

† Dentre essas atividades, as mais citadas foram: dança $(1,8 \%)$, alongamento $(1,7 \%)$, artes marciais $(1,2 \%)$ e hidroginástica $(1,1 \%)$; as demais foram praticadas por menos de $1 \%$ da amostra.

${ }^{\dagger}$ Among the activities, the most mentioned were: dancing (1.8\%), stretching (1.7\%), martial arts (1.2\%), and aqua aerobics (1.1\%); the remaining was practiced by less than $1 \%$ of the whole sample.

${ }^{\S}$ Esta variável teve 24 valores perdidos (1,9\% da amostra analisada). ${ }^{\varsigma}$ This variable had 24 missing values (1.9\% of the analyzed sample).

${ }^{\ddagger}$ Associação não mais significativa após a análise multivariável. ${ }^{\ddagger}$ These associations become no longer significant after multivariable analysis.

Paulo detectou que $63 \%$ dos adultos e idosos relataram praticar alguma atividade física como lazer ${ }^{15}$.

Dentre os principais resultados, encontrou-se que a caminhada foi a modalidade mais praticada pela maioria dos adultos, sendo referida por mais de $57 \%$ daqueles que realizaram alguma atividade física de lazer na última semana. Entre os idosos, por exemplo, 83\% relataram fazer caminhada. Tal achado é consistente com outros estudos que apontam ser a caminhada a atividade 
mais praticada pelos idosos ${ }^{2,16}$. Além dos benefícios que a caminhada pode trazer para a saúde, ela consiste em uma atividade de baixo custo e acessível para a maioria das pessoas, tanto que não houve diferença conforme o nível econômico. Outras atividades esportivas, como vôlei, tênis e natação, foram praticadas por menos de $1 \%$ dos entrevistados. Provavelmente porque não são acessíveis a grande parte da população e demandam recursos apropriados.

Um achado interessante revelado neste estudo indica que os indivíduos classificados como obesos possuem maior probabilidade de caminhar no lazer do que aqueles que estão com peso normal. Isso é o que se conhece por causalidade reversa. Ou seja, não significa que caminhar engorda, e sim que os indivíduos acima do peso praticam esta atividade mais frequentemente do que aqueles que estão com o seu peso normal. Acrescentando, um estudo conduzido em 2002 na mesma cidade em que foi feita esta pesquisa apontou que mais da metade dos adultos entrevistados consideram a caminhada como o melhor exercício para o emagrecimento ${ }^{17}$. Entretanto, muitas vezes a caminhada não chega a trazer benefícios por ser praticada em uma intensidade insuficiente para gerar adaptações fisiológicas. No presente estudo, cerca de $50 \%$ das pessoas que caminham relataram fazê-la numa intensidade leve, o que é um achado relevante para promover políticas de saúde pública mais consistentes.

Chama atenção também o fato de $91 \%$ dos adultos que praticam alguma atividade física de lazer estarem envolvidos apenas em uma atividade (geralmente caminhada). Exceção se atribui aos indivíduos praticantes de corrida ( $4 \%$ do total), em que mais da metade está engajada também em outras atividades. A maioria (56\%) dos praticantes de alguma atividade física de lazer referiu que o faz em uma intensidade moderada ou vigorosa. No entanto, apenas $30 \%$ atingiram a recomendação proposta para adultos e idosos $^{13}$, variando de $27 \%$ entre os que caminham a $56 \%$ entre os que correm. Quando considerado o tempo de prática, percebe-se que mais da metade (53\%) já possui o hábito de praticar atividade física, isto é, a pratica há mais de seis meses.

Quanto às características demográficas e socioeconômicas, foi possível identificar que cada modalidade possui um perfil diferente de adeptos. Quanto ao sexo do praticante, estudos nacionais confirmam que as mulheres são mais propensas do que os homens a se envolver em atividades como caminhada e ginástica, enquanto os homens se engajam mais frequentemente em esportes, corrida e musculação $0^{2,3}$. No entanto, destaca-se a participação das mulheres em algumas atividades que antes eram marcadas apenas pela presença masculina, como por exemplo o futebol e a musculação.

Com relação à idade, a frequência de todas as atividades físicas, exceto a caminhada, diminuiu consideravelmente entre os idosos. Mesmo estando mais sujeitos a problemas osteomusculares, apenas 1,5\% dos idosos disseram praticar musculação. Não se sabe até que ponto essa baixa prevalência se deve à falta de informação ou à falta de condições financeiras para frequentar uma academia. Estudos com abordagem qualitativa poderiam elucidar esta questão. Destaca-se que nenhum idoso relatou ter praticado corrida na semana anterior à entrevista. Um levantamento feito nas regiões Sudeste e Nordeste do país encontrou resultados semelhantes para a faixa etária, isto é, aumento na prevalência de caminhada entre os idosos, e redução na proporção engajada em atividades esportivas, de ginástica e de musculação² . $^{2}$

Cor da pele não apresentou associação significativa com nenhuma das modalidades analisadas. Isso poderia sugerir que não há discriminação racial no que concerne à prática desportiva. No entanto, não se pode esquecer que foram considerados apenas aqueles que praticam alguma atividade física de lazer, e alguns estudos já mostraram que a prática de atividade física é mais comum em indivíduos de cor de pele não-branca ${ }^{18}$. Quanto à situação conjugal, os que possuem companheiro(a) andam 
mais de bicicleta. Já os sem companheiro(a) praticam mais musculação, embora esta última associação tenha desaparecido na análise ajustada. Novamente, estudos com enfoque qualitativo se tornam importantes para explicar tais diferenças.

Pessoas com menos estudo foram mais propensas a caminhar ou andar de bicicleta, mesmo desconsiderando as atividades feitas por motivo de deslocamento. Isso se deve, provavelmente, ao fato de estas serem atividades mais comuns e acessíveis a esse grupo. A proporção de indivíduos com maior escolaridade engajados em outras atividades (além daquelas mais citadas) foi maior do que para os seus pares. Com relação ao nível econômico, os mais pobres andam mais de bicicleta, enquanto os mais ricos se envolvem mais frequentemente em atividades como musculação, ginástica e outros tipos de atividades menos comuns (danças, lutas etc.). Contudo, após ajustes para possíveis fatores de confusão, o nível econômico permaneceu (diretamente) associado apenas com a prática de musculação.

Por fim, os motivos que levam as pessoas a praticar atividade física também variam conforme a modalidade em que estão engajadas. Embora para quase todos os tipos de atividade a principal motivação seja melhorar a saúde, entre os praticantes de futebol, o principal motivo é o lazer ou a recreação. Isso poderia explicar o porquê de indivíduos com um mesmo perfil sociodemográfico praticarem atividades diferentes. Acrescentando, um estudo com adolescentes evidenciou que a prática de atividade física no lazer aumentou conforme o número de dias por semana em que o jovem se encontrava com amigos ${ }^{19}$. Isso demonstra que a rede social também pode interferir tanto no engajamento quanto na escolha das atividades esportivas.

Talvez a principal limitação deste estudo seja a utilização de um questionário não-validado. Entretanto, como foram consideradas apenas as atividades físicas realizadas na semana que antecedeu a entrevista, acredita-se que as respostas não tenham sido afetadas por um possível viés de informação, e que sejam condizentes com a realidade. Além disso, como as atividades feitas no lazer geralmente requerem intenção e planejamento, ao contrário daquelas feitas no trabalho ou no domicílio, o seu recordatório costuma ser mais preciso.

Concluindo, os resultados deste estudo apresentam dados inéditos para a região Sul do Brasil e poderão ser aplicados na elaboração de políticas de saúde pública que visem aumentar o nível de atividade física da população. Por exemplo, embora se saiba que os idosos representam a parcela populacional que proporcionalmente mais se beneficiaria com a prática de musculação, são justamente os que menos a praticam. A construção de pistas de caminhada ou ciclovias seria outra medida que, se implementada, poderia fazer com que mais pessoas aderissem à prática de atividade física, principalmente os extratos de menor nível socioeconômico. Desse modo, a junção de estratégias educacionais e ambientais se faz necessária, ainda mais se considerarmos que mais de um terço (38\%) da população investigada encontra-se no estágio de précontemplação para a prática de atividade física, isto é, não pratica e não tem intenção de praticar, conforme reportado em outro estudo pelo mesmo grupo de autores ${ }^{20}$.

Uma vez que a modalidade de atividade física praticada depende de diversos fatores locais, acredita-se que a proporção de indivíduos engajados em cada modalidade possa variar de região para região. Logo, os resultados encontrados no presente estudo podem não ser generalizáveis para outras regiões do país. Dessa forma, recomendase que sejam realizados outros estudos investigando o que as pessoas praticam em diferentes contextos, a fim de se poder estimar o efeito de variáveis ambientais e/ou de certos programas de intervenção em cada localidade (bairro, cidade ou Estado). Além disso, estudos longitudinais que avaliem a trajetória da atividade física ao longo da vida e a aderência a cada modalidade também são de grande relevância em termos de saúde pública. 


\section{Referências}

1. Craig CL, Marshall AL, Sjostrom M, Bauman AE, Booth ML, Ainsworth BE, et al. International physical activity questionnaire: 12-country reliability and validity. Med Sci Sports Exerc 2003; 35(8): 1381-95.

2. Monteiro CA, Conde WL, Matsudo SM, Matsudo VR, Bonsenor IM, Lotufo PA. A descriptive epidemiology of leisure-time physical activity in Brazil, 1996-1997. Rev Panam Salud Publica 2003; 14(4): 246-54.

3. Salles-Costa R, Heilborn ML, Werneck GL, Faerstein E, Lopes CS. Gênero e prática de atividade física de lazer. Cad Saúde Pública 2003; 19(sup. 2): 325-33.

4. Domingues MR, Barros AJ. Leisure-time physical activity during pregnancy in the 2004 Pelotas Birth Cohort Study. Rev Saúde Pública 2007; 41(2): 173-80.

5. Florindo AA, Latorre MRDO, Tanaka T, Jaime PC, Zerbini CAF. Fatores associados à prática de exercícios físicos em homens voluntários adultos e idosos residentes na Grande São Paulo, Brasil. Rev Bras Epidemiol 2001; 4(2): 105-13.

6. Hallal PC, Bertoldi AD, Goncalves H, Victora CG. Prevalência de sedentarismo e fatores associados em adolescentes de 10-12 anos de idade. Cad Saúde Pública 2006; 22(6): 1277-87.

7. IBGE. Censo Demográfico 2000. Rio de Janeiro: Instituto Brasileiro de Geografia e Estatística; 2000.

8. Barros AJD, Menezes AMB, Santos IS, Assunção MCF, Gigante DP, Fassa AG, et al. O Mestrado do Programa de Pós-graduação em Epidemiologia da UFPel baseado em consórcio de pesquisa: uma experiência inovadora. $R e v$ Bras Epidemiol 2008; 11(S1): 133-44.

9. ABEP. Critério Padrão de Classificação Econômica Brasil. São Paulo: Associação Brasileira de Empresas de Pesquisa; 2003.

10. Victora CG, Huttly SR, Fuchs SC, Olinto MT. The role of conceptual frameworks in epidemiological analysis: a hierarchical approach. Int J Epidemiol 1997; 26(1): 224-7.

11. Barros AJ, Hirakata VN. Alternatives for logistic regression in cross-sectional studies: an empirical comparison of models that directly estimate the prevalence ratio. BMC Med Res Methodol 2003; 3: 21.
12. Maldonado G, Greenland S. Simulation study of confounder-selection strategies. Am J Epidemiol 1993; 138(11): 923-36.

13. Haskell WL, Lee IM, Pate RR, Powell KE, Blair SN, Franklin BA, et al. Physical activity and public health: updated recommendation for adults from the American College of Sports Medicine and the American Heart Association. Circulation 2007; 116(9): 1081-93.

14. Pitanga FJ, Lessa I. Prevalência e fatores associados ao sedentarismo no lazer em adultos. Cad Saúde Pública 2005; 21(3): 870-7.

15. Monteiro CA, Moura EC, Jaime PC, Lucca A, Florindo AA, Figueiredo IC, et al. Monitoramento de fatores de risco para doenças crônicas por entrevistas telefônicas. Rev Saúde Pública 2005; 39(1): 47-57.

16. Almeida MMG, Moreira RF, Araújo TM, Pinho PS. Atividades de lazer entre idosos, Feira de Santana, Bahia. Rev Baiana Saúde Pública 2005; 29(2): 339-52.

17. Domingues MR, Araujo CL, Gigante DP. Conhecimento e percepção sobre exercício físico em uma população urbana do sul do Brasil. Cad Saúde Pública 2004; 20(1): 204-15.

18. Hallal PC, Victora CG, Wells JC, Lima RC. Physical inactivity: prevalence and associated variables in Brazilian adults. Med Sci Sports Exerc 2003; 35(11): 1894900.

19. Gonçalves H, Hallal PC, Amorim TC, Araujo CL, Menezes AM. Fatores socioculturais e nível de atividade física no início da adolescência. Rev Panam Salud Publica 2007; 22(4): 246-53.

20. Dumith SC, Gigante DP, Domingues MR. Stages of change for physical activity in adults from Southern Brazil: a population-based survey. Int J Behav Nutr Phys Act 2007; 4: 25.

Recebido em: 03/05/09

Versão final reapresentada em: 08/10/09

Aprovado em: 23/10/09 